NEUROLOGICAL PICTURE

\title{
Misdiagnosis of amyotrophic lateral sclerosis in a patient with dysphagia due to Chiari I malformation
}
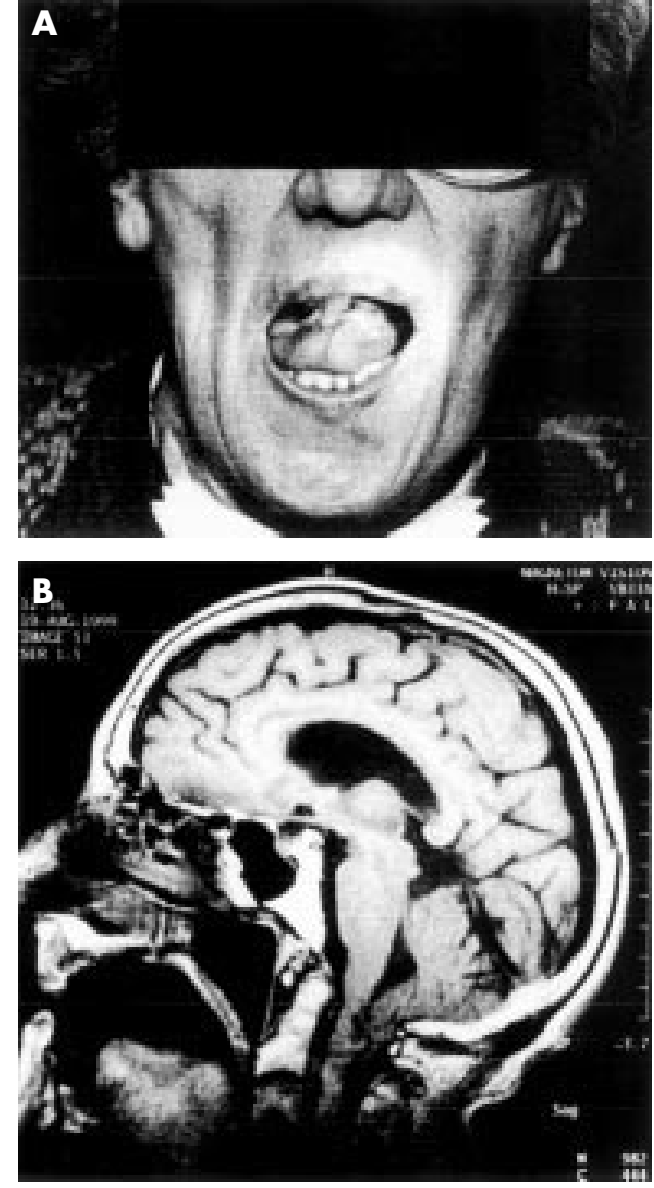

C

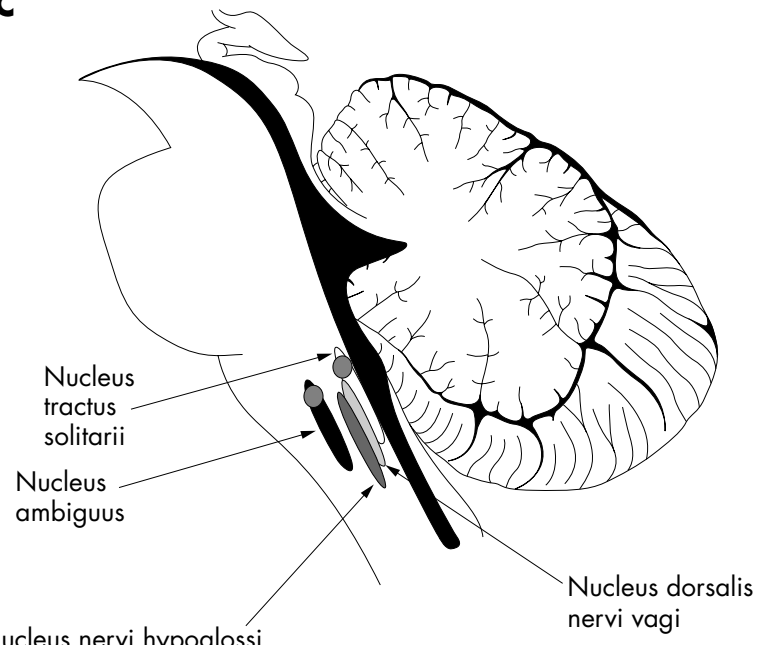

Dorsomedial and ventrolateral pattern generators

Figure 1 (A) Dysphagia with signs of aspirations of saliva extensive bilateral paresis, and atrophy of the tongue (with permission). (B) Brain MRI findings. (C) Semischematic drawing showing the site of regions involved in swallowing control in the upper medulla oblongata.
A 78 year old woman was admitted to our hospital's swallowing department because she had had progressive dysphagia for a year. Based on bilateral tongue atrophy with fibrillations and a normal CT, amyotrophic lateral sclerosis with bulbar onset had been diagnosed 1 month previously and a percutaneous gastrostomy was performed for feeding purposes.

The neurological examination on admission disclosed a dysphagia with signs of aspirations of saliva, extensive bilateral paresis, and atrophy of the tongue (fig l A) showing diffuse fibrillations. Further neurological examination was normal.

Somatosensory evoked potentials and nerve conduction studies as well as electromyography of various muscles of the upper and lower limbs showed no pathology. Comprehensive laboratory tests and CSF examination produced normal results. A videofluoroscopic swallowing study (VFSS) showed a reduced lingual control, especially for liquid consistencies, with subsequent predeglutitive aspiration. Brain and spinal MRI showed a Chiari I malformation with descent of the cerebellar tonsils to mid-level of the odontoid process (fig lB). There were no concomitant malformations such as syringomyelia or arachnoid cysts.

The patient underwent neurosurgical posterior fossa decompression. After the operation, weaning problems occurred and a tracheostomy was performed due to massive aspirations of saliva. The patient was readmitted to our hospital with a blocked tracheostomy tube. Oral feeding was not possible. After a 6 week period of swallowing therapy the dysphagia showed a marked improvement. Meanwhile partial feeding (about 70\%) is possible, the remainder being supplied by the gastrostomy tube. The tracheostomy tube has not yet been removed, as it is required to draw off bronchial mucus which occasionally causes respiratory problems. A follow up examination 6 months after the operation showed a bilateral reduction of the atrophy and the fibrillations of the tongue as well as improved tongue functions without aspirations on VFSS. There were still no signs of a generalised motor neuron disease.

Dysphagia may occur as the sole manifestation of adult Chiari I malformation. ${ }^{1}$ As far as we know, there has only been one case published with a Chiari I malformation in an adult mimicking a bulbar palsy in amyotrophic lateral sclerosis. ${ }^{2}$ This patient, however, did not show signs of lower motor neuron disease, which were predominant in our patient. We hypothesise that the dysphagia in our patient was caused by pressure exerted by the cerebellar mass on the hypoglossus nuclei and possibly also on the central pattern generators for swallowing situated in the dorsal region of the medulla (fig l C). A Chiari I malformation is an important differential diagnosis in patients with adult onset of dysphagia requiring MRI examination, even when presenting with "typical" lower motor neuron signs in bulbar muscles.

Authors' affiliations

M Paulig, M Prosiegel

Neurologisches Krankenhaus, Tristanstrasse 20, D-80804 Munich, Germany

Correspondence to: Dr M Paulig

\section{REFERENCES}

Achiron A, Kuritzky A. Dysphagia as the sole manifestation of adult type I Arnold-Chiari malformation. Neurology 1990;40:186-7.

2 Ikusaka M, Iwata M, Sasaki S, et al. Progressive dysphagia due to adult Chiari I malformation mimicking amyotrophic lateral sclerosis. J Neurol Neurosurg Psychiatry 1996;60:357-8. 